\title{
Sindecanos: Su potencial uso diagnóstico y pronóstico en cáncer de próstata
}

\author{
HÉCTOR R. CONTRERAS
}

\section{Syndecans in the diagnosis and prognosis of prostate cancer}

Syndecans, a family of heparan sulphate proteoglycans that are present in the cell surface are involved in the control of cell proliferation, apoptosis and transformation. Syndecans 1 and 2 have a central role in processes such as position control, invasion, angiogenesis and metastases of several types of cancer. The expression of Syndecan 1 in epithelial cells, decreases when cells are transformed and acquire invasive properties. This decreased expression is associated to a bad prognosis. Syndecan 2, originally described in mesenchymal cells, favors cell apoptosis, increases angiogenesis and controls the death of cancer cells subjected to chemotherapy. Both syndecans are present in basal and epithelial cells of prostate cancer. Their lower expression is associated to more undifferentiated tumors. Disturbances in the expression and subcellular location of syndecans predict the relapse of localized tumors. Syndecans 1 and 2 can be considered tumor suppression genes and can be targets for new treatments. The detection of circulating fragments of these molecules could be useful for the early detection of prostate cancer.

(Rev Med Chile 2010; 138: 95-101).

Key words: Neoplasm invasiveness; Prostatic neoplasms; Proteoglycans.

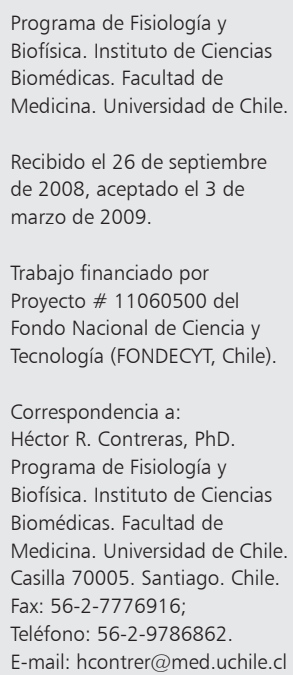

E 1 potencial invasivo y metastático de las células cancerosas está modulado por una serie de ligandos extracelulares provenientes desde la matriz extracelular (MEC) y que además pueden controlan la proliferación celular, la dinámica del citoesqueleto, la apoptosis y la expresión génica. Muchas de estas acciones son mediadas por un conjunto de moléculas denominadas moléculas de adhesión celular (MAC). Estas son proteínas de membrana que actúan como un enlace entre el exterior y el interior de la célula. Entre las moléculas de adhesión podemos mencionar, la superfamilia de moléculas similares a inmunoglobulinas, cadherinas, integrinas, proteoglucanos de heparán sulfato, selectinas, receptores de ácido hialurónico y otras ${ }^{1}$. Los fenómenos más importantes en que están involucradas las moléculas de adhesión en la progresión maligna incluyen: sobrevivencia independiente de adhesión a la $\mathrm{MEC}^{2}$, migración, invasión, metástasis ${ }^{3}$ y angiogénesis ${ }^{4,5}$. El estudio de las moléculas de adhesión en cáncer de próstata, en el contexto del estudio de otras moléculas, tiene una importancia no sólo pronóstica, sino que puede ser el objetivo de nuevas estrategias terapéuticas y predictores de respuesta a terapia ${ }^{6}$.

\section{Proteoglucanos de heparansulfato: Sindecanos}

Los proteoglucanos (PG) de la superficie celular son un importante grupo de moléculas que participan en la adhesión celular y motilidad. Algunos promueven la adhesión celular, a través de la unión con proteínas estructurales de la MEC (laminina, fibronectina, colágeno, etc), la unión de factores de crecimiento, quimoquinas y citoquinas y finalmente la adhesión célula-célula a través del reconocimiento de MAC (selectinas, CAMs, etc). 
Sindecanos: Su potencial uso diagnóstico y pronóstico en cáncer de próstata - H. Contreras

Los PG que recientemente se han involucrado en la progresión tumoral, debido a su participación en la modulación de la adhesión y movilidad celular son los sindecanos ${ }^{7,8}$. Los sindecanos son una familia de proteoglucanos que contienen cadenas de glucosaminoglucano de heparán sulfato unidos a un esqueleto proteico central (core protéico). Esta glucoproteína presentan 3 dominios, uno extracelular, que posee uniones de glucosaminoglucanos de heparán sulfato (asociado a elementos de la matriz extracelular), otro de transmembrana (responsable de la fijación a la membrana plasmática) y uno intracelular (asociado a rutas de señalización) (Figura 1). Existen 4 tipos de sindecanos descritos ${ }^{9}$, expresados en diferentes tipos celulares los cuales varían su expresión en ciertas condiciones patológicas (progresión tumoral, invasión y metástasis) además de participar en procesos de señalización intracelular ${ }^{10}$. De los cuatro miembros de la familia de los sindecanos, se considera que los sindecanos -1 y -3 y los -2 y -4 forman subfamilias, basándose en la comparación de sus secuencias aminoacídicas ${ }^{11}$. La mayoría de las células expresa combinaciones distintas de los cuatro sindecanos en la medida que se diferencian, resultando en patrones selectivos de expresión que probablemente reflejan distintas funciones. El sindecano-1 se expresa típicamente en células epiteliales ${ }^{12}$, el sindecano- 3 en células neuronales ${ }^{13}$, el sindecano-2 se encuentra principalmente en células mesenquimales ${ }^{14}$, en tanto el sindecano-4 es ubicuo ${ }^{15,16}$. Los sindecanos se localizan en la membrana plasmática y funcionan como receptores de superficie en la regulación de transducción de señales dependiente de adhesión durante el crecimiento celular, adhesión celular, migración y organización del citoesqueleto, a través de la unión a la MEC y ligandos solubles ${ }^{17}$. Su función estaría dada no sólo como receptores de membrana sino también al interactuar con otras moléculas de adhesión, cambiando su afinidad de unión a ligandos y, por consecuencia, modulando vías de señalizaciones distintas pero sobrepuestas. Estas vías de señalización estarían determinadas en última instancia por las regiones variables del dominio citoplasmático a través de proteínas de la familia ERM (ezrina-radixina-moesina) ${ }^{18}$. También sus funciones pueden estar determinadas por el core proteico del correspondiente sindecano expresado por cada célula en particular ${ }^{10}$ (Figura 2). El funcionamiento de los sindecanos en la superficie celular es estructurando complejos multiméricos en dominios específicos de la membrana plasmática. Como parte de estos complejos, el core proteico de los sindecanos, tiene la capacidad de dimerizarse y generar respuestas funcionales tan especializadas como la maduración dendrítica a través de la fosforilación del receptor tirosina quinasa EphB2 ${ }^{19}$.

\section{Sindecanos y cáncer}

La participación de los sindecanos en cáncer ha sido estudiada principalmente respecto del sindecano-1. A pesar de que la mayoría de las evidencias apuntan a un papel como supresor de tumores ${ }^{20-23}$, se ha visto una expresión elevada de este proteoglucano en tumores de mama ${ }^{24}$, páncreas ${ }^{25}$ y metástasis óseas de tumores hematológicos y de mama ${ }^{26}$. El sindecano-1 se encuentra altamente expresado en la cara basolateral de las

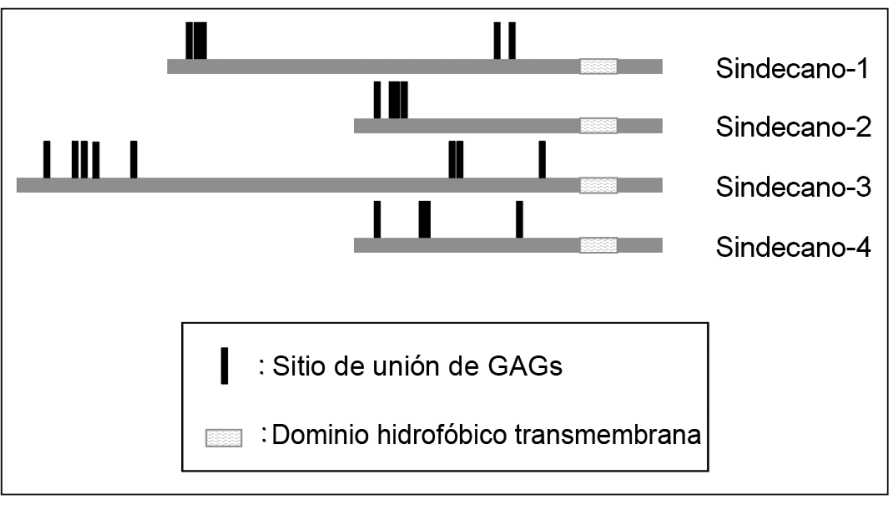

Figura 1. Representación esquemática de la organización de los dominios de los sindecanos. Las barras verticales indican los sitios de unión de $\mathrm{GAG}^{64}$. 


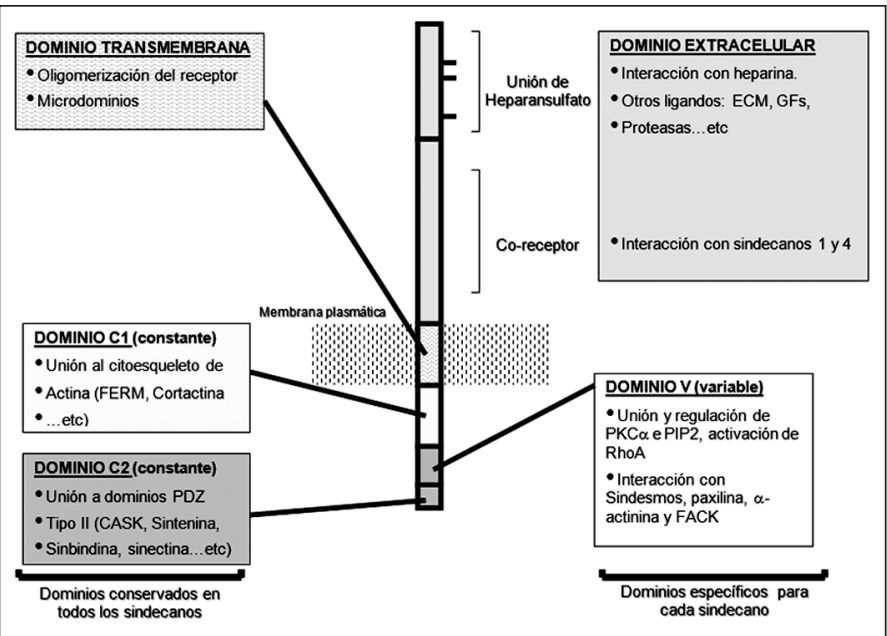

Figura 2. Esquema que representa las diferentes acciones intracelulares asociadas a los dominios intracelular (C1, V y C2) y extracelular (verde) de los sindecanos ${ }^{65}$. células epiteliales, donde a través de su dominio citoplasmático interactúa con el citoesqueleto de actina, modulando la adhesión celular y la señalización de factores de crecimiento ${ }^{12}$. En el cáncer de próstata, se han presentado datos contradictorios, por un lado se ha encontrado que la sobreexpresión de sindecano-1 puede predecir la recurrencia temprana de la enfermedad y está significativamente asociada con la sobrevida específica del tumor, el alto grado de Gleason y la sobre expresión de Ki67 y Bcl-227; por otro lado Kiviniemi et al, 2004 reportan una expresión alterada de sindecano- 1 en cáncer de próstata y una relación inversa entre la expresión de sindecano-1 y el grado de

Tabla 1. Evidencias de expresión de sindecano 1 y 2 en diferentes tipos de cáncer

\begin{tabular}{|c|c|c|}
\hline $\begin{array}{l}\text { Tipo de } \\
\text { Sindecano }\end{array}$ & Observación & Referencias \\
\hline \multirow[t]{8}{*}{ Sindecano-1 } & Expresión detectada por IHQ se asocia con tumores indiferenciados en Ca cabeza y cuello & 57 \\
\hline & En Ca mama expresión es dependiente de E-cadherina & 34 \\
\hline & Ectodominio puede ser liberado por clivaje proteolítico y participa en crecimiento celular & 58 \\
\hline & En CaP se altera expresión y existe correlación inversa con score Gleason & 28,36 \\
\hline & Se asocia a factores de crecimiento tipo FGF y la progresión de tumores de próstata & 59 \\
\hline & Expresión alterada en CaP & $27,28,35$ \\
\hline & Ectodominio presente en Ca pulmón y biopsias gliomas & 60 \\
\hline & Existe valor pronóstico en cáncer de próstata & 52 \\
\hline \multirow[t]{8}{*}{ Sindecano-2 } & Expresado principalmente en células estromales & 98 \\
\hline & Participa en organización del citoesqueleto células tumorales & 44 \\
\hline & Se sobreexpresa en células normalmente migratorias & 62 \\
\hline & $\begin{array}{l}\text { Expresión en líneas celulares de Ca colon se asocia disminución expresión E-cadherina e } \\
\text { induce fenotipo migratorio }\end{array}$ & 41 \\
\hline & Interactúa con factores angiogénicos (interleukinas, VEGF, bFGF y TGF $\beta$ ) & 36,63 \\
\hline & Regula los procesos angiogénicos en células endoteliales de gliomas & 55 \\
\hline & Afecta la apoptosis inducida por quimioterapia en osteosarcoma & 56 \\
\hline & Suprime la activación de metaloproteinasas de matriz & 51 \\
\hline
\end{tabular}


Gleason del tumor, encontrándose similar relación con respecto de la expresión de citoqueratinas de alto peso molecular utilizada como un marcador de células del epitelio basal ${ }^{28}$. Otros estudios sobre sindecano- 1 en células tumorales de mieloma múltiple evaluado en geles de colágeno confirman sus propiedades adhesivas y anti invasivas ${ }^{29}$. Entre los cambios en la expresión de sindecanos se ha descrito que eventualmente el dominio extracelular (ectodominio) de estas moléculas puede ser liberado intacto por actividad proteolítica de metaloproteinasas (sheeding) pudiendo ser evaluado en plasma sanguíneo. Recientemente, para el cáncer de pulmón, se reportó una correlación positiva respecto del sheeding de sindecano-1 y el grado de diferenciación del tumor ${ }^{30}$ y con menor variaciones plasmáticos en cáncer de cuello ${ }^{31}$ y linfomas de Hodgkin ${ }^{32}$. Esta expresión diferencial, estaría relacionada con la disminución de E-cadherina. En estudios experimentales de transformación epitelio-mesenquimal, la expresión de sindecano-1 se asocia con la mantención de la morfología epitelial, apreciándose una pérdida conjunta de la expresión de sindecano-1 con E-cadherina durante trasformación epitelio-mesenquimal, procesos habituales durante etapas del desarrollo embrionario $^{33}$. Asimismo, otro estudio realizado en células tumorales mamarias plantea que la disminución de sindecano-1 ocurriría por un mecanismo dependiente de E-cadherina ${ }^{34}$. Sindecano-1 cambia su patrón de expresión durante la progresión de varios tipos de cánceres, incluido el prostático, sugiriéndose una utilidad como marcador del grado de diferenciación tumoral ${ }^{35,36}$. En el cáncer prostático, sindecano-1 tendría una utilidad pronóstica, sugiriéndose como marcador de malignidad, ya que en estudios inmunohistoquímicos la expresión de sindecano- 1 se encuentra alterada en el cáncer prostático y que existe una correlación inversa entre el grado de Gleason y la expresión de sindecano-1, observándose una menor expresión a mayor grado de malignidad histológica ${ }^{28,37,38}$.

Para el sindecano-2 la información descrita lo involucra centralmente como responsable de características migratorias y fenotípicas, clásicas de una célula tumoral. En células mesenquimales su sobre expresión induce estructuras tipo filopodios y lamelipodios con actividad cdc42Hs y a través de ezrina (de la familia de la proteínas ERM) se une al citoesqueleto de actina ${ }^{18,39,40}$. La asociación existente entre sindecano- 2 y su participación en la transformación tumoral y oncología molecular principalmente se centra en estudios utilizando líneas celulares de cáncer de colon y pulmón ${ }^{41,42}$. En células de cáncer de colon el sindecano-2 media la adhesión y proliferación ${ }^{42}$, su expresión induce un fenotipo migratorio ${ }^{41}$ y su bloqueo con cDNA antisense de sindecano-2 reduce la actividad tumorigénica de estas células ${ }^{43}$. En líneas de cáncer pulmonar, se ha visto que el sindecano- 2 participa en la organización del citoesqueleto de actina ${ }^{44}$, actuando en conjunto con sindecano- $4{ }^{45}$. Por otro lado, sindecano-2 es determinante en las adhesiones focales estimuladas por kinasas y que establecen las células de fibrosarcomas durante su migración ${ }^{46}$ También se ha descrito que a través de su dominio intracelular y, señalizando a través de JNK, induce apoptosis cuando es sobre-expresado en células de osteosarcoma ${ }^{47}$ y que participaría en la transformación oncogénica vía activación de $\mathrm{ras}^{48}$. Recientemente, se ha reportado que sindecano-2 estaría participando en procesos de angiogénesis actuando como un co-receptor para el EVGF ${ }^{49}$. En células de colon su sobreexpresión revierte el fenotipo tumoral, disminuyendo su capacidad invasiva y modificando sus propiedades adhesivas. Se sugiere una participación directa de sindecano-2 en la transición epitelio-mesénquima modificándose las propiedades morfológica, adhesivas e invasivas en células epiteliales de cáncer de colon. La participación de sindecano-2 en cáncer de próstata no ha sido aún reportada. Resultados recientemente publicados utilizando inmunohistoquímica sobre tejidos de prostatectomías parciales, indican la presencia de sindecano-2 en las células epiteliales prostáticas en glándulas intactas, disminuyendo su expresión en estrecha relación cuanto menos diferenciado es la región tumoral cuando es evaluada por el índice de Gleason ${ }^{50}$. Los cambios en la expresión de sindecano-2, se encontraría asociada a la disminución de la expresión de E-cadherina, molécula de adhesión que clásicamente otros autores estudian en tumores de origen epitelial ${ }^{6}$. La participación de sindecano-2 en procesos de transformación maligna adquiere mayor importancia cuando recientemente se describe su rol en la supresión de la activación de metaloproteinasas de matriz con la consiguiente inhibición de la invasión y metástasis ${ }^{51}$.

Recientemente, en estudios prospectivos se logró determinar que la expresión de sindecano-1 en biopsias de pacientes con prostatectomías radi- 
cales se asocia estrechamente con la recurrencia de cáncer prostático evaluado con la presencia de PSA plasmático ${ }^{52}$. Estudios similares no publicados, demuestran que sindecano - 1 y -2 , evaluados en biopsias de pacientes con cáncer clínicamente localizado, logran predecir significativamente la recidiva bioquímica del cáncer de próstata ${ }^{53}$. En este reciente estudio, no sólo la expresión de ambos sindecanos adquiere importancia, sino que también la distribución en la expresión, por lo que no sólo la información cuantitativa adquieres relevancia, sino que también la información cualitativa.

\section{Implicancias en diagnóstico y pronóstico}

Para los sindecanos los hallazgos reportados en los últimos años se focalizan principalmente en la participación en procesos de angiogénesis, metástasis y control de la proliferación celular ${ }^{55}$. Más recientemente, la capacidad del sindecano-2 de inducir muerte celular por apoptopsis en poblaciones celulares sometidas a quimioterapia, hacen pensar que la utilización de terapias combinadas podrían dar mejores resultados en cánceres clínicamente localizados ${ }^{56}$. Si a ello le agregamos la capacidad demostrada de sindecano-2 de inhibir la activación de metaloproteinasas de matriz, podrían sugerir que su sobreexpresión inhibiría la capacidad invasiva y metastásica de las células en aquellos cánceres localizados ${ }^{51}$. Un dato importante es que la capacidad angiogénica de los sindecanos ha sido detectada sólo cuando se produce la liberación del ectodominio del proteoglucano en el proceso denominado sheeding ${ }^{55}$. Si se confirma que este sheeding es un evento temprano en la transformación oncogénica, su evaluación en el plasma podría ser un elemento muy importante en la detección precoz del cáncer. Respecto del cáncer prostático, las recientes implicancias en la detección de sindecanos-1 y -2, ofrece insospechadas proyecciones. La información confirma una estrecha asociación entre expresión de sindecano 1 con el riesgo de reaparición de la enfermedad en pacientes con adenocarcinoma clínicamente localizado ${ }^{52}$. En Chile, se han reportado hallazgos similares para sindecano 1 y 2 , en pacientes con residiva bioquímica de cáncer prostático. Por lo tanto, considerando que la liberación del ectodominio de los sindícanos- 1 y - 2 es un proceso temprano en la transformación oncogénica y que ambos pueden ser detectados en el plasma, los sindecanos-1 y -2 podrían ser considerados como moléculas que sumadas al PSA podrían contribuir a una mejor detección precoz del cáncer y ser utilizados como marcadores tumorales de recidiva bioquímica en pacientes con la enfermedad localizada.

Basado en las diferentes funciones descritas, los sindecanos pueden ser considerados como potenciales blancos para el desarrollo de nuevas terapias en el tratamiento del cáncer y otras alteraciones fisiológicas.

\section{Referencias}

1. Lukas Z, Dvorák K. Adhesion molecules in biology and oncology. Acta Vet Brno 2004; 73: 93-104.

2. Frisch SM, Screaton RA. Anoikis mechanisms. Curr Opin Cell Biol 2001; 13: 555-62.

3. Liotta LA, Kohn EC. Invasion and metastases. In: Cancer Medicine. $6^{\text {a }}$ Ed. Hamilton (Canada). BC Decker Inc. 2003 [en línea: http://www.ncbi.nlm.nih.gov/entrez/ query.fcgi? $\mathrm{cmd}=$ Search \&db=books\&doptcmdl $=\mathrm{GenB}$ ookHL\&term $=$ Biology + of + Prostate + ancer + AND $+\mathrm{cm}$ ed6\%5Bbook $\% 5$ D+AND+355280\%5Buid $\% 5$ D \& rid $=c$ med6.section.27977].

4. Gasparini G. The rationale and future potential angiogenesis inhibitors neoplasia. Drugs 1999; 58: 17-38.

5. Cao Y. Antiangiogenic cancer therapy. Sem Cancer Biol 2004; 14: 139-145.

6. Quinn D, Henshall S, Sutherland R. Molecular markers of prostate cancer outcome. Eur J Cancer 2005; 41: 85887.

7. Sanderson R. Heparan sulfate proteoglycans in invasion and metastasis. Sem Cell Dev Biol 2001; 12: 89-98.

8. Sasisekharan R, Shriver Z, Venkataraman G, Narayanasami U. Roles of heparan-sulphate glycosaminoglycans in cancer. Nat Rev Cancer 2002; 2: 521-8.

9. Woods A, Couchman J. Syndecans: synergistic activators of cell adhesion. Trends Cell Biol 1998; 8: 189-92.

10. Beauvais D, Rapraeger A. Syndecans in tumor cell adhesion. Reprod Biol Endocrinol 2004; 2: 1-12.

11. Oh E, Couchman JR. Syndecans-2 and -4; close cousins, not identical twins. Mol Cells 2004; 17: 181-7.

12. Rapraeger A, Jalkanen M, Bernfield M. Cell surface proteoglycan associates with the cytoskeleton at the basolateral cell surface of mouse mammary epithelial cells. J Cell Biol 1986; 103: 2683-96.

13. Chernousov MA, Carey D. N-Syndecan (syndecan-3) from neonatal rat brain binds basic fibroblast growth factor. J Biol Chem 1993; 268: 16810-4. 
14. Klass C, Couchman J, WoodS A. Control of extracellular matrix assembly by syndecan-2 proteoglycan. J Cell Biol 1999; 144: 575-86.

15. Baciu P, Goetinck P. Protein kinase $\mathrm{C}$ regulates the recruitment of syndecan-4 into focal contacts. Mol Biol Cell 1995; 6: 1503-13.

16. Han I, Park H, Oh E. New insights into syndecan-2 expression and tumorigenic activity in colon carcinoma cells. J Mol Histol 2004; 35: 319-26.

17. Carey DJ. Syndecans: multifunctional cell-surface coreceptors. Biochem J 1997; 327: 1-16.

18. Granés F, García R, Casaroli-Marano RP, Castel S, Rocamora N, Reina M, et al. Syndecan-2 induces filopodia by active cdc42Hs. Exp Cell Res 1999; 248: 439-56.

19. Ethell IM, Irie F, Kalo MS, Couchman JR, Pasquale EB, Yamaguchi Y. EphB/syndecan-2 signaling in dendritic spine morphogenesis. Neuron 2001; 31: 1001-13.

20. Bayer-Garner IB, Dilday B, Sanderson RD, Smoller BR. Syndecan-1 expression is decreased with increasing aggressiveness of basal cell carcinoma. Am J Dermopathol 2000; 22: 119-22.

21. Aref S, Goda T, El-Sherbiny M. Syndecan-1 in multiple myeloma: relationship to conventional prognostic factors. Hematology 2003; 8: 221-8.

22. Li HG, Xie DR, Shen XM, Li HH, Zeng H, Zeng YJ. Clinicopathological significance of expression of paxilin, syndecan-1 and EMMPRIN in hepatocellular carcinoma. World J Gastroenterol 2005; 11: 1445-51.

23. Langford JK, Yang Y, Kieber-Emmons T, Sanderson RD. Identification of an invasion regulatory domain within the core protein of syndecan-1. J Biol Chem 2005; 280: 3467-73.

24. Tsanou E, Ioachim E, Briasoulis E, Charchanti A, Damala $\mathrm{K}$, Karavasilis V, et al. Clinicopathological study of the expression of syndecan-1 in invasive breast carcinomas. Correlation with extracellular matrix components. J Exp Clin Cancer Res 2004; 23: 641-50.

25. Conejo JR, Kleeff J, Koliopanos A, Matsuda K, Zhu ZW, Goecke H, et al. Syndecan-1 expression is up-regulated in pancreatic but not in other gastrointestinal cancers. Int J Cancer 2000; 88: 12-20.

26. Sanderson RD, Yang Y, Suva LJ, Kelly T. Heparan sulfate proteoglycans and heparanase partners in osteolytic tumor growth and metastasis. Matrix Biol 2004; 23: 341-52.

27. Zellweger T, Ninck C, Mirlacher M, Annefeld M, Glass A, Gasser T, et al. Tissue microarray analysis reveals prognostic significance of syndecan-1 expression in prostate cancer. Prostate 2003; 55: 20-9.

28. Kiviniemi J, Kallajoki M, Kujala I, Matikainen M, Alanen $\mathrm{K}$, Jalkanen M, et al. Altered expression of syndecan-1 in prostate cancer. APMIS 2004; 112: 89-97.
29. Liu W, Litwack D, Stanley M, Langford K, Lander A, Sanderson R. Heparan sulfate proteoglycans as adhesive and anti-invasive molecules. J Biol Chem 1988; 273: 22825-32.

30. Endo K, Takino T, Miyamori H, Kinsen H, Yoshizaki T, Furukawa M, et al. Cleavage of syndecan-1 by membrane type matrix metalloproteinase-1 stimulates cell migration. J Biol Chem 2003; 278: 40764-70.

31. Anttonen A, Leppa S, Heikkila P, Grenman R, Joensuu $\mathrm{H}$ Effect of treatment of larynx and hypopharynx carcinomas on serum syndecan-1 concentrations. J Cancer Res Clin Oncol 2006; 132: 451-7.

32. Vassilakopoulos TP, Kyrtsonis MC, Papadogiannis A, Nadali G, Angelopoulou MK, Tzenou T, et al. Serum levels of soluble syndecan-1 in Hodgkin's lymphoma. Anticancer Res 2005; 25 (6C): 4743-6.

33. Sun D, Mcalmon K, Davies J, Bernfield M, Hay E. Simultaneous loss of expression of syndecan-1 and E-cadherin in the embryonic palate during epithelial-mesenchymal transformation. Int J Dev Biol 1998; 42: 733-6.

34. Leppa S, Vleminckx K, Van Roy F, Jalkanen M. Syndecan-1 expression in mammary epithelial tumor cells is E-cadherin-dependent. J Cell Sc 1996; 1393-403.

35. Mennerich D, Vogel A, Klaman I, Dahl E, Lichtener R, Rosenthal A, et al. Shift of syndecan-1 expression from epithelial to stromal cells during progression of solid tumours. Eur J Cancer 2004; 40: 1373-82.

36. Chen D, Adenekan B, Chen L, Vaughan D, Gerald W, Feng Z, et al. Syndecan-1 expression in locally invasive and metastatic prostate cancer. Urology 2004; 63: 402-7.

37. Etzioni R, Hawley S, Billheimer D, True Ld, Knudsen B. Analyzing patterns of staining in immunohistochemical studies: application to a study of prostate cancer recurrence. Cancer Epidemiol Biomarkers Prev 2005; 14: 1040-6.

38. Kambham N, Kong C, Longacre Ta, Natkunam Y. Utility of syndecan-1 (CD138) expression in the diagnosis of undifferentiated malignant neoplasms: a tissue microarray study of 1,754 cases. Appl Immunohistochem Mol Morphol 2005; 13: 304-10.

39. Granés F, Urena JM, Rocamora N, Vilaró S. Ezrin links syndecan-2 to the cytoskeleton. J Cell Sci 2000; 113 (Pt 7): 1267-76.

40. Granés F, Berndt C, Roy C, Mangeat P, Reina M, Vilaró S. Identification of a novel Ezrin-binding site in syndecan-2 cytoplasmic domain. FEBS Lett 2003; 547: 212-6.

41. Contreras HR, Fabre M, Granes F, Casaroli-Marano R, Rocamora N, Herreros AG, et al. S Syndecan-2 expression in colorectal cancer-derived HT-29 M6 epithelial cells induces a migratory phenotype. Biochem Biophys Res Commun 2001; 286: 742-51. 
42. Park H, Kim Y, Lim Y, Han I, Oh ES. Syndecan-2 mediates adhesion and proliferation of colon carcinoma cells. J Biol Chem 2002; 277: 29730-6.

43. Kim Y, Park H, Lim Y, Han I, Kwon HJ, Woods A, et al. Decreased syndecan-2 expression correlates with trichostatin-A induced-morphological changes and reduced tumorigenic activity in colon carcinoma cells. Oncogene 2003; 22: 826-30.

44. Munesue S, Kusano Y, Oguri K, Itano N, Yoshitomi Y, Nakanishi $\mathrm{H}$, et al. The role of syndecan-2 in regulation of actin-cytoskeletal organization of Lewis lung carcinoma-derived metastatic clones. Biochem J 2002; 363: 201-9.

45. Kusano Y, Yoshitomi Y, Munesue S, Okayama M, Oguri K. Cooperation of syndecan-2 and syndecan-4 among cell surface heparan sulfate proteoglycans in the actin cytoskeletal organization of Lewis lung carcinoma cells. J Biochem (Tokyo) 2004; 135: 129-37.

46. Park H, Han I, Kwon HJ, Oh ES. Focal adhesion kinase regulates syndecan-2-mediated tumorigenic activity of HT1080 fibrosarcoma cells. Cancer Res 2005; 65: 9899905.

47. Modrowski D, Orosco A, Thevenard J, Fromigue O, Marie PJ. Syndecan-2 overexpression induces osteosarcoma cell apoptosis: Implication of syndecan-2 cytoplasmic domain and JNK signaling. Bone 2005; 37: 180-9.

48. Huang JW, Chen CL, Chuang NN. Trap RACK1 with Ras to mobilize Src signaling at syndecan-2/p120-GAP upon transformation with oncogenic ras. Biochem Biophys Res Commun 2005; 330: 1087-94.

49. Essner JJ, Chen E, Ekker SC. Syndecan-2. Int J Biochem Cell Biol 2006; 38: 152-6. Epub 2005 Sep 15.

50. Contreras HR, Ledezma R, Vergara J, Cifuentes F, Barra C, Gallegos I, et al. 2008. The expression of Syndecan-1 and 2 is associated with Gleason score and epithelialmesenchymal transition markers E-cadherin and betacatenin, in prostate cancer. Urol Oncol 2009; Published ahead of print DOI: 10.1016/j.urolonc.2009.03.018.

51. Munesue S, Yoshitomi Y, Kusano Y, Koyama Y, Nishiyama A, Nakanishi $\mathrm{H}$, et al. A novel functions of syndecan-2, suppression of matrix metalloproteinase-2 activation, which causes suppression of metastasis. J Biol Chem 2007; 282: 28164-74. Epub 2007 Jul 10.

52. Shariat SF, Svatek RS, Kabbani W, Walz J, Lotan Y, Karakiewicz PI, et al. Prognostic value of syndecan-1 expression in patients treated with radical prostatectomy. BJU Int 2008; 101: 232-7. Epub 2007 Sep 13.
53. Ledezma R, Cifuentes F, Gallegos I, Fullá J, Huidobro C, Castellón E, Contreras HR. Shifit of expression pattern of syndecan 1 and 2 predicts biochemical recurrence in prostate cancer. Urol Oncol 2009 (en revisión editorial).

54. Fears CY, Gladson CL, Woods A. Syndecan-2 is expressed in the microvasculature of gliomas and regulates angiogenic processes in microvascular endothelial cells. J Biol Chem 2006; 281: 14533-6. Epub 2006 Mar 30.

55. Orosco A, Fromigué O, Bazille C, Entz-Werle N, Levillain P, Marie PJ, et al. Syndecan-2 affects the basal and chemotherapy-induced apoptosis in osteosarcoma. Cancer Res 2007; 67: 3708-15.

56. Inki P, Joensuu H, Grénman R, Klemi P, Jalkanen M. Association between syndecan-1 expression and clinical outcome in squamous cell carcinoma of the head and neck. Br J Cancer 1994; 70: 319-23.

57. Fitzgerald ML, Wang Z, Park PW, Murphy G, Bernfield M. Shedding of syndecan- 1 and -4 ectodomains is regulated by multiple signaling pathways and mediated by a TIMP-3-sensitive metalloproteinase. J Cell Biol 2000; 148: 811-24.

58. Wu X, Kan M, Wang F, Jin C, Yu C, Mckeehan WL. A rare premalignant prostate tumor epithelial cell syndecan-1 forms a fibroblast growth factor-binding complex with progression-promoting ectopic fibroblast growth factor receptor 1. Cancer Res 2001; 61: 5295-302.

59. Seidel C, Sundan A, Hjorth M, Turesson I, Dahl IM, Abildgaard N, et al. Serum syndecan-1: a new independent prognostic marker in multiple myeloma. Blood 2000; 95: 388-92.

60. Rapraeger AC, Ott VL. Molecular interactions of the syndecan core proteins. Curr Opin Cell Biol 1998; 10: 620-8. Review.

61. Kusano Y, Oguri K, Nagayasu Y, Munesue S, Ishihara M, Saiki I, et al. Participation of syndecan 2 in the induction of stress fiber formation in cooperation with integrin alpha5beta1: structural characteristics of heparan sulfate chains with avidity to $\mathrm{COOH}$-terminal heparin-binding domain of fibronectin. Exp Cell Res 2000; 256: 434-44.

62. Halden Y, Rek A, Atzenhofer W, Szilak L, Wabnig A, Kungl AJ. Interleukin-8 binds to syndecan-2 on human endothelial cells. Biochem J 2004; 377 (Pt 2): 533-8.

63. Zimmermann P, David G. The syndecans, tuners of transmembrane signaling. FASEB J 1999; 13 Suppl: S91-S100.

64. Beauvais DM, Rapraeger AC. Syndecans in tumor cell adhesion and signaling. Reprod Biol Endocrinol 2004; 2: 3. Review. 